\title{
Properties of a double layer with asymmetric electrolytes: ion-penetrable membrane carrying nonuniformly distributed fixed charges
}

\author{
Jyh-Ping Hsu *, Yung-Chih Kuo \\ Department of Chemical Engineering, National Taiwan University, Taipei, Taiwan 10617, ROC
}

Received 21 December 1994; revised 30 May 1995; accepted 1 June 1995

\begin{abstract}
We consider a planar, cylindrical, or spherical particle immersed in an arbitrary a:b electrolyte solution. The particle comprises an ion-impenetrable, rigid uncharged core and an ion-penetrable membrane carrying nonuniformly distributed fixed charges. This model simulates a wide class of biocolloids and particles covered with an artificial surface layer. Two classes of nonuniform fixed charge distributions, one linear and the other nonlinear, are examined. These distributions all have the same average or apparent space charge density, which is independent of the geometry of the particle. On the basis of a perturbation method, the distribution of the gradient of the electrical potential of the system under consideration is derived, and the essential thermodynamic properties of the electrical double layer are estimated. The result of numerical simulation reveals that the degree of accuracy of the present semi-analytical treatment is satisfactory. We show that the distribution of fixed charges in membrane phase has a significant effect on the thermodynamic properties of the system under consideration. Assuming that the fixed charges are distributed homogeneously may lead to an appreciable deviation.
\end{abstract}

Keywords: Electric double layer; Asymmetric electrolytes; Ion-penetrable membrane; Thermodynamic properties; Nonuniform fixed charge distribution

\section{Introduction}

The electrical potential distribution for a system comprises a charged surface immersed in an electrolyte solution is governed by the Poisson-Boltzmann equation [1]. Although asymmetric electrolytes are common in practice, the discussions about the solution of this equation are almost always limited to symmetric electrolytes. For asymmetric electrolytes, the PoissonBoltzmann equation is highly nonlinear, and solving it analytically is almost impossible, except under extreme

\footnotetext{
* Corresponding author.
}

conditions, such as a simple geometry at a low surface potential. In a recent study, the difficulty of solving a nonlinear Poisson-Boltzmann equation was alleviated by resorting to a semi-empirical procedure for rigid planar surfaces immersed in an a:b electrolyte solution [2]. The basic thermodynamic properties predicted by the approximate method were reasonably accurate. The analysis was extended to the case of a particle coated with a membrane bearing fixed charges [3]. This is of particular significance for biocolloids and some particles coated with an artificial membrane. In these cases, the classic rigid surface model is insufficient for the description of their behavior in an electrolyte solution. 
The analysis in [3] was based on the assumption that the fixed charges in membrane are uniformly distributed (independent of position). Although this assumption simplifies the mathematical treatment, a generalization to nonuniformly distributed fixed charges is highly desirable. This is because that membranes may exhibit microscopic and macroscopic structural inhomogeneities in practice [4]. Typical example includes perfluorosulphonate materials which possess microstructural inhomogeneity [5]. The inhomogeneity nature of a membrane leads to nonuniform distributed fixed charges, which can have a significant effect on its physicochemical properties $[4,6,7]$. Reiss and Bassignana [7], for instance, suggested that the inhomogeneity of the fixed charge distribution in an ion exchange membrane might be the origin of its superselectivity. In a study of the ion transport in ion exchange membranes, Selvey and Reiss [8] found that inhomogeneously distributed fixed charges may result in an increase in current efficiency. It was shown that the electrophoretic mobility of a particle coated with an ion-penetrable membrane is highly correlated with the fixed charge distribution in the membrane phase [9]. Clearly, a realistic model should take the structural characteristics of a membrane into account. The aim of the present study is to extend the analysis of [3]. In particular, the effect of nonuniformly distributed fixed charges in a membrane on its thermodynamic properties is investigated.

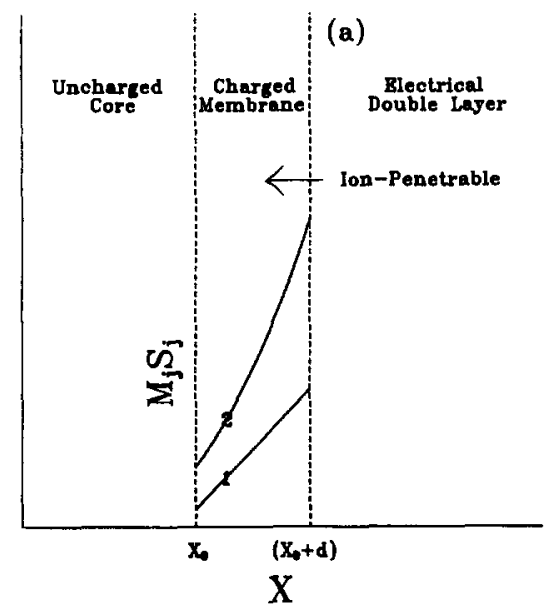

\section{Theory}

A schematic representation of the system under consideration is illustrated in Fig. 1. A rigid surface immersed in an a:b electrolyte solution is coated with an ion-penetrable membrane of dimensionless thickness $d$. The region $X<X_{0}$ denotes the uncharged core of a particle, $X_{0}<X<\left(X_{0}+d\right)$ is the ion-penetrable membrane, and $X>\left(X_{0}+d\right)$ represents the liquid phase, $X$ being a dimensionless distance. Let $N(X)$ be the dimensionless distribution of the fixed charges in the membrane. For convenience, we assume that these fixed charges are negative. Depending upon the nature of membrane, various types of distribution for $N(X)$ can be assumed. For illustration, we consider a class of linear distributions $N_{1}(X)$, and a class of nonlinear distributions $N_{2}(X)$. The mathematical treatment, however, does not limit the choice of $N(X)$. The definitions of $N_{1}(X)$ and $N_{2}(X)$ are

$$
\begin{aligned}
& N_{1}=\left(Z N_{0} N_{\mathrm{A}} / a n_{\mathrm{a}}^{0}\right)\left[1+\alpha\left(X-X_{0}\right)\right] / R_{1} \\
& N_{2}=\left(Z N_{0} N_{\mathrm{A}} / a n_{\mathrm{a}}^{0}\right)\left\{1+\exp \left[\alpha\left(X-X_{0}\right)\right]\right\} / R_{2}
\end{aligned}
$$

where $R_{1}$ and $R_{2}$ are defined in Appendix A. In these expressions, $\alpha$ is a parameter characterizing the fixed charge distribution, $N_{0}$ and $Z$, are, respectively, the average space density and the valence of the charged groups in the membrane $\left(-Z e N_{0} N_{\mathrm{A}}\right.$ is the average density of the fixed charges, $e$ being the elementary

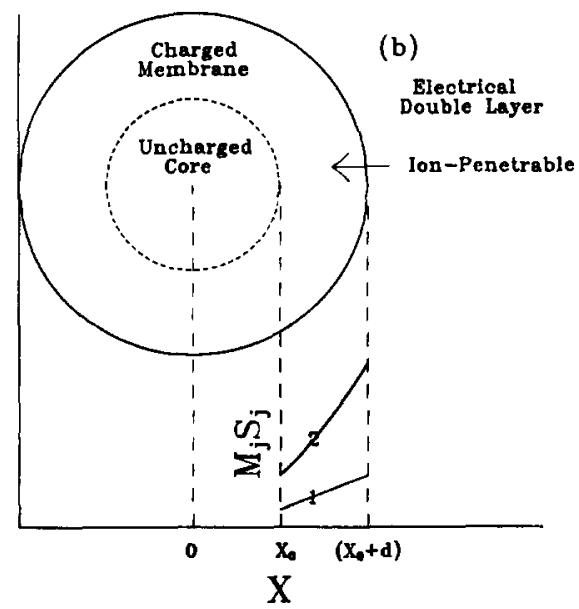

Fig. 1. A schematic representation of the system under consideration: $d$ is the dimensionless thickness of the membrane. The region $X<X_{0}$ denotes the uncharged core of a particle, $X_{0}<X<\left(X_{0}+d\right)$ represents the ion-penetrable membrane, and $X>\left(X_{0}+d\right)$ is the liquid phase: (a) planar surface $(m=0)$; (b) cylindrical $(m=1)$ and spherical $(m=2)$ surfaces. Curve 1, linear fixed charge distribution. Curve 2, nonlinear fixed charge distribution. 

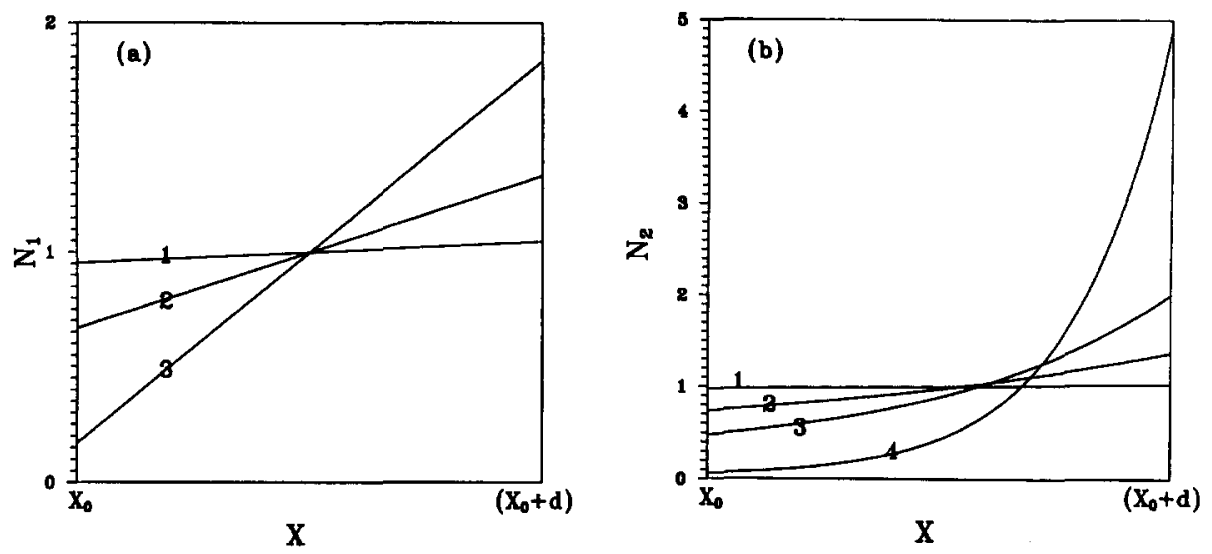

Fig. 2. Variation in the dimensionless fixed charge distribution in the membrane. The parameters used are: $a=1, Z=1, N_{0}=10^{-3} \mathrm{M}, n_{\mathrm{a}}^{0} /$ $N_{\mathrm{A}}=10^{-3} \mathrm{M}, m=0, d=1$ : (a) linear distribution with $\alpha=0.1$ (curve 1), $\alpha=1$ (curve 2), $\alpha=10$ (curve 3); (b) exponential distribution with $\alpha=0.1$ (curve 1), $\alpha=1$ (curve 2), $\alpha=2$ (curve 3), $\alpha=5$ (curve 4).

charge), $N_{\mathrm{A}}$ represents the Avogadro number, and $n_{\mathrm{a}}^{0}$ is the number concentration of cation in the bulk liquid phase. As shown in Appendix A, the amount of fixed charges per unit area of membrane (average or apparent surface charge density) is independent of the type of fixed charge distribution, the value of $\alpha$, and the type of surface. Some other properties of $N_{1}$ and $N_{2}$ are also summarized in Appendix A. As illustrated in Fig. 2, Eqs. (1a) and (1b) predict that the fixed charges are more concentrated near the membrane-liquid interface than in the interior for a positive $\alpha$. This is consistent with the observation of Pineri [6].

A general expression for the distribution of the electrical potential for planar, cylindrical, and spherical surfaces can be written as

$\frac{1}{X^{m}} \frac{\mathrm{d}}{\mathrm{d} X}\left(X^{m} \frac{\mathrm{d} \psi}{\mathrm{d} X}\right)=\frac{g+i N_{j}}{a+b}, i=0,1, j=1,2$

where $\quad \psi=e \phi / k_{\mathrm{B}} T, \quad g=[\exp (b \psi)-\exp (-a \psi)]$, $X=\kappa r$, and $\kappa^{2}=e^{2} a(a+b) n_{\mathrm{a}}^{0} / \epsilon_{0} \epsilon_{\mathrm{r}} k T$. In these expressions, $\phi$ is the electrostatic potential, $\epsilon_{\mathrm{r}}$ and $\epsilon_{0}$ are, respectively, the relative permittivity of the system and the permittivity of a vacuum, $\kappa$ and $k_{\mathrm{B}}$ are the reciprocal Debye length and the Boltzmann constant, respectively, $T$ is the absolute temperature, $r$ represents the distance, $i$ is a region index ( $i=0$ for double layer or $X>X_{0}+d, i=1$ for membrane or $X_{0}<X<\left(X_{0}+d\right)$ ), $j$ is an index characterizing the type of fixed charge distribution $\left(N_{1}\right.$ or $\left.N_{2}\right)$, and $m$ is a geometry parameter ( $m=0$ for a flat plate, $m=1$ for a cylinder, $m=2$ for a sphere).
The boundary conditions associated with Eq. (2) are

$$
\begin{aligned}
& \psi \rightarrow 0 \text { and }(\mathrm{d} \psi / \mathrm{d} X) \rightarrow 0 \text { as } X \rightarrow \infty \\
& \psi_{X \rightarrow\left(X_{0}+d\right)^{-}}=\psi_{X \rightarrow\left(X_{0}+d\right)^{+}}=\psi_{\mathrm{d}} \\
& (\mathrm{d} \psi / \mathrm{d} X)_{X \rightarrow\left(X_{0}+d\right)^{-}}=(\mathrm{d} \psi / \mathrm{d} X)_{X \rightarrow\left(X_{0}+d\right)^{+}} \\
& \psi \rightarrow \psi_{\mathrm{c}} \text { and } \mathrm{d} \psi / \mathrm{d} X \rightarrow 0 \text { as } X \rightarrow X_{0}
\end{aligned}
$$

In these expressions $\psi_{\mathrm{c}}$ and $\psi_{\mathrm{d}}$ are, respectively, the dimensionless electrical potential at the coremembrane interface and that at the membrane-liquid interface. If $d$ is sufficiently large, the Donnan equilibrium can be reached. In this case, the following boundary condition needs to be satisfied:

$\psi \rightarrow \psi_{\text {Don }}$ and $\frac{1}{X^{m}} \frac{\mathrm{d}}{\mathrm{d} X}\left(X^{m} \frac{\mathrm{d} \psi}{\mathrm{d} X}\right) \rightarrow 0$ as $X \rightarrow X_{0}$

where $\psi_{\text {Don }}$ is the dimensionless Donnan potential.

\subsection{Planar surface}

For a planar geometry, $r$ represents the horizontal distance, and the core-membrane interface is located at $r_{0}$. The distribution of the potential gradient in double layer is obtained by integrating Eq. (2) with $i=0$ and employing Eq. (2a). We have

$$
\begin{array}{r}
\mathrm{d} \psi / \mathrm{d} X=-\operatorname{Sgn}(\psi)[2 /(a+b)]^{1 / 2}\{(1 / b)[\exp (b \psi) \\
-1]+(1 / a)[\exp (-a \psi)-1]\}^{1 / 2}
\end{array}
$$


Suppose that the linear size of the membrane phase of a particle is relatively smaller than that of the uncharged core, i.e. $X_{0}$ is sufficiently large. In this case, the distribution of the potential gradient in membrane phase can be estimated by employing the perturbation method invoked by Overbeek et al. [10]. This method suggests that $\mathrm{d} \psi / \mathrm{d} X$ can be approximated by

$\mathrm{d} \psi / \mathrm{d} X=H(\psi, X)=\sum_{i=0}^{n} e_{i}(\psi) / X^{i}$

In practice, choosing $n$ as 2 will be sufficiently accurate [11]. Expanding $N_{1}$ and $N_{2}$ into negative power series in $X$ around $\left(X_{0}+d / 2\right)$, we have

$N_{j}=\sum_{i=0}^{n} w_{j, i} / X^{i}, j=1,2$

where $w_{j, i}$ are summarized in Appendix B. Substituting Eq. (4) into Eq. (2) with $i=1$ yields

$H\left(\frac{\partial H}{\partial \psi}\right)_{X}+\left(\frac{\partial H}{\partial X}\right)_{\psi}=\frac{g+N_{j}}{a+b}, X_{0}<X<X_{0}+d$

Substituting Eqs. (4) and (5) into Eq. (6) and collecting terms of the same order in $X$, lead to the set of equations below:

$e_{0} e_{0}^{\prime}=\left(g+w_{j, 0}\right) /(a+b)$

$e_{1} e_{0}^{\prime}+e_{0} e_{1}^{\prime}=\left(w_{j, 1}\right) /(a+b)$

$\cdots$

where the differentiation is with respect to $\psi$. The associated boundary conditions are deduced from Eq. (2d) as

$e_{i} \rightarrow 0$ as $\psi \rightarrow \psi_{c}\left(\right.$ or $\left.X \rightarrow X_{0}\right), i=0,1,2, \ldots, n$

Solving Eqs. (7a), (7b),..., subject to Eq. (7c) gives the variation of function $H(\psi, X)$ or $\mathrm{d} \psi / \mathrm{d} X$ across the membrane. It can be shown that

$\mathrm{d} \psi / \mathrm{d} X=e_{0}+e_{1} / X+\ldots$

where

$e_{0}=[2 /(a+b)]^{1 / 2}\{(1 / b)[\exp (b \psi)$

$\left.-\exp \left(b \psi_{\mathrm{c}}\right)\right]+(1 / a)[\exp (-a \psi)-\exp ($

$\left.\left.\left.-a \psi_{\mathrm{c}}\right)\right]+w_{j, 0}\left(\psi-\psi_{\mathrm{c}}\right)\right\}^{1 / 2}$

$e_{1}=\left[w_{j, 1}\left(\psi-\psi_{\mathrm{c}}\right)\right] /\left[(a+b) e_{0}\right]$
The Donnan potential can be calculated by Eqs. (2) and $(2 \mathrm{e})$. These equations yield

$g\left(\psi_{\text {Don }}\right)+N_{j}\left(X_{0}\right)=0$

An iterative procedure is available to the resolution of this type of equation [3]. The relation between $\psi_{\mathrm{u}}$ and $\psi_{\text {Don }}$ is determined by Eqs. (3) and (8) with Eqs. (2b) and $(2 \mathrm{c})$. We obtain the following approximate result:

$$
\begin{aligned}
\psi_{\mathrm{d}}=\psi_{\text {Don }}+\frac{X_{0}+d}{w_{j, 0}\left(X_{0}+d\right)+w_{j, 1}}\left\{( 1 / b ) \left[\exp \left(b \psi_{\text {Don }}\right)\right.\right. \\
\left.-1]+(1 / a) \times\left[\exp \left(-a \psi_{\text {Don }}\right)-1\right]\right\}
\end{aligned}
$$

\subsection{Cylindrical and spherical surfaces}

In this case, $r$ is either the distance measured from the axis of a cylinder or that measured from the center of a sphere. The perturbation method similar to that employed for the case of a planar surface is adopted to estimate the variation of the potential gradient in double layer. Here, we assume that $X_{0}$ is large enough so that this method is applicable to Eq. (2) with $i=0$. On the basis of Eqs. (2) and (2a), it can be shown that

$$
\begin{aligned}
& \mathrm{d} \psi / \mathrm{d} X=[2 /(a+b)]^{1 / 2}\{(1 / b)[\exp (b \psi)-1] \\
& +(1 / a)[\exp (-a \psi)-1]\}^{1 / 2}-\frac{1}{X} \frac{2 m}{a} \tanh (a \psi / 4) \\
& +\frac{1}{X^{2}}\left[\frac{m^{2} \tanh ^{2}(a \psi / 4)-4 m(m-1) \ln [\cosh (a \psi / 4)]}{a k_{3} \sinh (a \psi / 2)}\right] \\
& X>\left(X_{0}+d\right)
\end{aligned}
$$

where $k_{3}$ is defined in Eq. (C15). Similarly, the distribution of potential gradient in membrane takes the form (Appendix C)

$\frac{\mathrm{d} \psi}{\mathrm{d} X}=d_{j, 0}+\frac{d_{j, 1}}{X}+\frac{d_{j, 2}}{X^{2}}+\ldots, X_{0}<X<\left(X_{0}+d\right)$

where $d_{j, i}, i=0,1,2, \ldots, j=1,2$, are given in Appendix C.

The Donnan potential is described by Eq. (9), and the relation between $\psi_{\mathrm{d}}$ and $\psi_{\text {Don }}$ can be determined by Eqs. (12), (2d) and (2e). We have

$d_{0}\left(\psi_{\text {Don }}\right)+\frac{d_{1}\left(\psi_{\text {Don }}\right)}{X_{0}}+\frac{d_{2}\left(\psi_{\text {Don }}\right)}{X_{0}^{2}}+\ldots=0$ 
where $d_{j, i}$ is abbreviated as $\mathrm{d}_{i}$. It can be shown that an approximate solution to Eq. (13) is (Appendix D)

$$
\begin{aligned}
& \psi_{d} \simeq \psi_{\text {Don }}+\left(1 / w_{j, 0}\right)\left\{( 1 / b ) \left[\exp \left(b \psi_{\text {Don }}\right)\right.\right. \\
& -1]+(1 / a)\left[\exp \left(-a \psi_{\text {Don }}\right)-1\right] \\
& \left.-\frac{a+b}{2}\left[\frac{d_{1}\left(\psi_{\text {Don }}\right)_{2}}{X_{0}}+\frac{d_{2}\left(\psi_{\text {Don }}\right)_{2}}{X_{0}^{2}}\right]^{2}\right\}
\end{aligned}
$$

where $d_{1}\left(\psi_{\text {Don }}\right)_{2}$ and $d_{2}\left(\psi_{\text {Don }}\right)_{2}$ are defined in Appendix D.

\subsection{Double-layer properties}

Following the same treatment as that employed for the case of uniformly distributed fixed charges [3], we assume that the total or apparent charge density $\sigma_{\mathrm{t}}$ ( $=-Z e N_{0} N_{\mathrm{A}} d / \kappa$, Appendix A) comprises two parts: an equivalent charge density due to the charges distributed in membrane $\sigma_{s 1}$, and the equivalent charge density at membrane-liquid interface, $\sigma_{\mathrm{d} 1}$. The former is the charge density defined by distributing all the charges in the membrane over a surface located somewhere in the range $\left[X_{0}, X_{0}+d\right]$. We have

$$
\sigma_{\mathrm{t}}=\sigma_{\mathrm{d} 1}+\sigma_{\mathrm{s} 1}
$$

Define the dimensionless interface charge density $p_{d 1}$ as

$p_{\mathrm{d} 1}=-a e \sigma_{\mathrm{d} 1} / 2 k_{3} \epsilon_{0} \epsilon_{\mathrm{r}} k_{\mathrm{B}} T \kappa$,

It can be shown that, regardless of the value of $m$,

$$
\sigma_{\mathrm{d} 1}=-\epsilon_{0} \epsilon_{\mathrm{r}} \frac{\kappa k_{\mathrm{B}} T}{e}\left(\frac{\mathrm{d} \psi}{\mathrm{d} X}\right)_{X=X_{0}+d}
$$

Eqs. (16a), (16) and (11) lead to

$$
\begin{aligned}
p_{\mathrm{d} 1}=\frac{a}{2 k_{3}}\left(\frac{\mathrm{d} \psi}{\mathrm{d} X}\right)_{X}=X_{0}+d & =-\sinh \left(a \psi_{\mathrm{d}} / 2\right) \\
& -\frac{1}{X_{0}+d} \frac{m}{k_{3}} \tanh \left(a \psi_{\mathrm{d}} / 4\right)+\frac{1}{\left(X_{0}+d\right)^{2}}
\end{aligned}
$$

$\frac{m^{2} \tanh ^{2}\left(a \psi_{\mathrm{d}} / 4\right)-4 m(m-1) \ln \left[\cosh \left(a \psi_{\mathrm{d}} / 4\right)\right]}{2 k_{3} \sinh \left(a \psi_{\mathrm{d}} / 2\right)}$

\subsection{Helmholtz free energy}

The Helmholtz free energy $F_{\mathrm{e} 1}$ comprises the free energy due to the double layer, $F_{\mathrm{e} 1 \mathrm{~d}}$, and that due to the membrane, $F_{\mathrm{els}}$

$F_{\mathrm{e} 1}=F_{\mathrm{e} 1 \mathrm{~d}}+F_{\mathrm{e} 1 \mathrm{~s}}$

Here, $F_{\text {eld }}$ is the equivalent electrical work done by adding gradually the same amount, but opposite in sign, of the excess charges in the liquid phase to membraneliquid interface, and $F_{\mathrm{e} 1 \mathrm{~s}}$ is that done by adding the total amount of charges in the membrane phase to coremembrane interface. We have

$$
\begin{aligned}
& F_{\mathrm{c} 1 \mathrm{~d}}=\int_{0}^{\sigma_{\mathrm{d} 1}} \phi\left(\sigma_{\mathrm{d} 1}^{\prime}\right) d \sigma_{\mathrm{d} 1}^{\prime} \\
& F_{\mathrm{e} 1 \mathrm{~s}}=\int_{\sigma_{\mathrm{d} 1}}^{\sigma_{\mathrm{t}}} \phi_{\mathrm{Don}}\left(\sigma_{\mathrm{t}}^{\prime}\right) \mathrm{d} \sigma_{\mathrm{t}}^{\prime}
\end{aligned}
$$

where $\phi_{\mathrm{d}}$ and $\phi_{\mathrm{Don}}$ are the potential at $X=X_{0}+d$ and the Donnan potential, respectively. Employing Eqs. (9), (14), and (16b) gives

$$
\begin{aligned}
& F_{\mathrm{eld}}=\left(\frac{2 k_{\mathrm{B}} T}{a e}\right)^{2} \epsilon_{0} \epsilon_{\mathrm{r}} \kappa k_{3}\left\{\left[p_{\mathrm{d} 1} \ln \left(p_{\mathrm{d} 1}+q_{\mathrm{d} 1}\right)\right.\right. \\
& \left.-q_{\mathrm{d} 1}+1\right]-\frac{m}{k_{3}\left(X_{0}+d\right)} \ln \left(\frac{q_{\mathrm{d} 1}+1}{2}\right) \\
& +\frac{1}{\left(X_{0}+d\right)^{2}}\left[\frac{m}{2 k_{3}^{2}} \frac{\left(q_{\mathrm{d} 1}-1\right)\left(q_{\mathrm{d} 1}+2\right)}{q_{\mathrm{d} 1}\left(q_{\mathrm{d} 1}+1\right)} \quad(17 \mathrm{c})\right. \\
& \left.\left.-\frac{m(m-1)}{2 k_{3}^{2}} \int_{v}^{1} \frac{\ln Z_{1}}{Z_{1}-1} \mathrm{~d} Z_{1}\right]\right\} \\
& F_{\mathrm{el} 1 \mathrm{~s}}=-\sigma_{\mathrm{d} 1}\left(\frac{2 k_{\mathrm{B}} T}{a e}\right) \ln Y_{\mathrm{Don}}+d \kappa^{-1} n_{\mathrm{a}}^{0} k_{\mathrm{B}} T \\
& \times\left[2 \ln Y_{\mathrm{Don}}+Y_{\mathrm{Don}}^{-2}+\frac{2}{k-2} Y_{\mathrm{Don}}^{-2}-Y_{\mathrm{Don}}^{-2}-\frac{2}{k-2} Y_{\mathrm{Don}}^{k-2}\right] \\
& \int_{v}^{1} \frac{\ln Z_{1}}{Z_{1}-1} \mathrm{~d} Z_{1}=\sum_{i=1}^{\infty} \frac{v^{2}}{i^{2}}-\frac{\pi^{2}}{6}+\ln (v) \ln (1-v) \\
& \text { where } \quad q_{\mathrm{d} 1}=\left(p_{\mathrm{d} 1}^{2}+1\right)^{1 / 2}, \quad v=\left[2 /\left(q_{\mathrm{d} 1}+1\right)\right],
\end{aligned}
$$


$Y=\exp (a \psi / 2)$, and $k$ is defined in Appendix C. $Y_{\text {Don }}^{\prime}$ is the value of $Y$ calculated at the Donnan potential in which the total surface charge density is regarded as $\sigma_{\mathrm{d} 1}$, not $\sigma_{\mathrm{t}}$, i.e. $Y_{\text {Don }}^{\prime}=Y_{\text {Don }}\left(\sigma_{\mathrm{d} 1}\right)$.

\subsection{Amount of ion adsorption}

From the thermodynamic point of view, the electrical Helmholtz free energy can be evaluated by

$\mathrm{d} F_{\mathrm{el}}=-\Gamma_{+} \mathrm{d} \mu_{+}-\Gamma_{-} \mathrm{d} \mu_{-}$

where $\Gamma_{+}$and $\Gamma_{-}$are the amount of adsorbed counterions and that of co-ions, respectively, $\mu_{+}$and $\mu_{-}$are the corresponding chemical potentials, which are functions of $\kappa$. Applying the electroneutrality condition leads to [3]

$\Gamma \equiv \Gamma_{-}=-2 \kappa^{-1} n_{\mathrm{a}}^{0} N_{\mathrm{A}}{ }^{-1} k_{3} D_{\mathrm{d} 1}-\frac{2}{k N_{\mathrm{A}}}\left(\frac{\sigma_{\mathrm{s} 1}}{a e}\right.$

$\left.+\frac{\kappa}{2 k_{\mathrm{B}} T} \frac{\partial F_{\mathrm{els}}}{\partial \kappa}\right)$

where $D_{\mathrm{d} 1}$ and $\partial F_{\mathrm{e} \mid \mathrm{s}} / \partial \kappa$ are defined in Appendix E.

\subsection{Entropy}

The entropy of a double layer $S_{\mathrm{e} 1}$ can be calculated by

$S_{\mathrm{e} 1}=\left(\partial F_{\mathrm{e} 1} / \partial T\right)_{n_{i}}$

Substituting Eq. (17) into this expression yields

$S_{\mathrm{e} 1}=\frac{-3}{2} \frac{F_{\mathrm{e} 1 \mathrm{~d}}}{T}-\left(\frac{k_{\mathrm{B}}}{a e}\right)^{2} 2 T \epsilon_{0} \epsilon_{\mathrm{r}} \kappa k_{3}\left\{p_{\mathrm{d} 1} \ln \left(q_{\mathrm{d} 1}-p_{\mathrm{d} 1}\right)\right.$

$+\frac{m}{k_{3}\left(X_{0}+d\right)}$

$\times \frac{q_{\mathrm{d} 1}-1}{q_{\mathrm{d} 1}}-\frac{1}{\left(X_{0}+d\right)^{2}}\left[\frac{m^{2}}{k_{3}^{2}}\left(\frac{2}{q_{\mathrm{d} 1}\left(q_{\mathrm{d} 1}+1\right)}\right.\right.$

$\left.-\frac{1}{q_{\mathrm{d} 1}^{2}}\right)+\frac{m(m-1)}{k_{3}^{2}}$

$\left.\left.\times \frac{\ln \left(\left(q_{\mathrm{d} 1}+1\right) / 2\right)}{q_{\mathrm{d} 1}}\right]\right\}$

$$
-2 \sigma_{\mathrm{d} 1}\left(k_{\mathrm{B}} / a e\right) \ln Y_{\text {Don }}^{\mathrm{N}}+d \kappa^{-1} n_{a}{ }^{0} k_{\mathrm{B}}
$$

$\times\left[2 \ln Y_{\text {Don }}+Y_{\text {Don }}^{-2}+\frac{2}{k-2}\right.$

$$
\left.-Y_{\text {Don }}^{k-2} Y_{\text {Don }}^{\prime-2}+\frac{2}{k-2} Y_{\text {Don }}^{k-2}\right]
$$

\section{Discussion}

In the present analysis it is assumed that the dielectric constant of the membrane phase is the same as that of the liquid phase for simplicity. If the difference between these constants is appreciable, the ion partitioning effect which arises from the Born energy needs to be considered. It was shown that the effect of ion partitioning can have a significant influence on the distributions of potential and ions [12].

Note that $e_{1}$ must satisfy the condition $e_{1}\left(\psi_{\mathrm{c}}\right)=0$. This can be shown by applying the L'Hopital's rule on Eq. (8b) and employing Eq. (7a) to yield

$\lim _{\psi \rightarrow \psi_{c}} e_{1}=\lim _{\psi \rightarrow \psi_{c}} w_{j, 1}\left(\frac{e_{0}}{g+w_{j, 0}}\right)$

According to Eq. (9), $g\left(\psi_{\mathrm{c}}\right)+w_{j, 0} \simeq 0$. Since $e_{0}\left(\psi_{\mathrm{c}}\right)=0$ by Eq. (8a), Eq. (22) leads to $e_{1}\left(\psi_{\mathrm{c}}\right)=0$.

As in the case of uniformly distributed fixed charges [3], the exact values of the Helmholtz free energy and the entropy of the double layer are not defined. However, the exact value of the amount of ion adsorption $\Gamma_{i}$ can be calculated by

$\Gamma_{i}=\int_{V}\left(n_{i}-n_{i}^{0}\right) \mathrm{d} \overrightarrow{\mathbf{r}} / A N_{\mathrm{A}}$

where $n_{i}$ and $n_{i}^{0}$ are, respectively, the number concentration of species $i$ and that of species $i$ in the bulk liquid phase, $\vec{r}$ represents the position vector, $V$ is the volume of the system, and $A$ denotes the surface area of membrane. After replacing $\boldsymbol{n}_{i}$ with the corresponding Boltzmann distribution, and solving Eq. (2) for the potential distribution, Eq. (23) can be integrated numerically. Fig. 3 shows the variation in the amount of ion adsorption as a function of parameter $k$ for the case where the fixed charges are linearly distributed; the corresponding result for the case of nonlinearly distributed fixed charges is presented in Fig. 4. Both the approximate values based on the present analysis and the exact numerical values are presented in these figures. The average deviation in the amount of ion adsorption is summarized in Table 1. Fig. 3 and Fig. 4 and Table 1 reveal that the deviation of the present approximate result, Eq. (19), from the corresponding exact value, estimated by Eq. (23), is less than $5 \%$. 


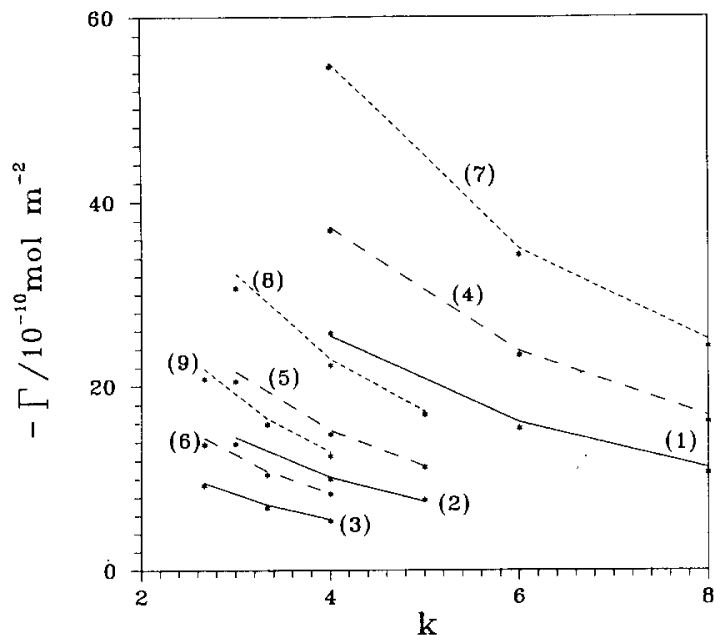

Fig. 3. Variation in the amount of ion adsorption as a function of parameter $k$ for the case the fixed charges are linearly distributed with $\alpha=1$ : (-) $m=0$; (-) $m=1 ;(-) m=2 . a=1$ in curves 1,4 , and $7 ; a=2$ in curves 2,5 , and $8 ; a=3$ in curves 3,6 , and $9, a$ being the valence of the cation for an $a: b$ electrolyte. *, approximate results predicted by the present analysis, Eq. (19). Key: $Z=1, N_{0}=10^{-3}$ $\mathrm{M}, d=1$, ionic strength $=10^{-3} \mathrm{M}, T=298.15 \mathrm{~K}$, and $\epsilon_{\mathrm{r}}=78 . X_{0}=10$ for planar surface, and $X_{0}=2$ for cylindrical and spherical surfaces.

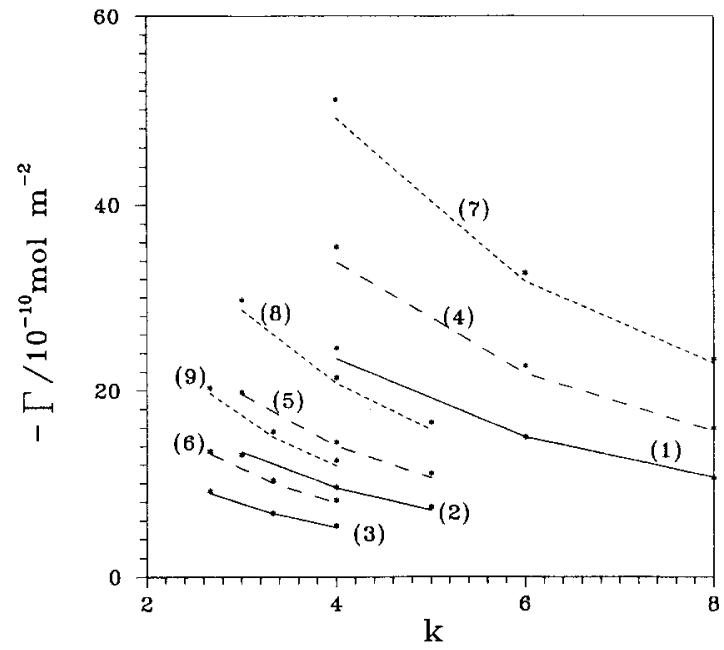

Fig. 4. Variation in the amount of ion adsorption as a function of parameter $k$ for the case the fixed charges are exponentially distributed. The parameters and the symbols used are the same as Fig. 3, except that $\alpha=0.8$.

For a nonplanar surface, the dimensionless thickness of the uncharged core of a particle $X_{0}$ is assumed to be large enough. The effect of the magnitude of $X_{0}$ on the performance of the present method is illustrated in Fig. 5. As can be seen from this figure, the result pre- dicted by the present method is satisfactory for $X_{0}>2$. This condition is usually satisfied in practice.

The distribution of electrical potential in membrane for a planar surface can be calculated through an iterative procedure. Integrating Eq. (2) subject to Eqs. $(2 a)-(2 c)$, we obtain

$$
\begin{aligned}
& (\mathrm{d} \psi / \mathrm{d} X)^{2}=[2 /(a+b)]\{(1 / b)[\exp (b \psi) \\
& \left.-1]+(1 / a)[\exp (-a \psi)-1]+\int_{0}^{\psi} N_{j}(X) \mathrm{d} \psi\right\}
\end{aligned}
$$

Table 1

Average percent deviation in the amount of ion adsorption for the case of Fig. 3 and Fig. 4. The percent deviation is defined as $100 \% \times \mid$ (present approximate result - exact value)/exact value $\mid$. The present approximate result is obtained by Eq. (19), and the exact value is calculated by Eq. (23). Here, $m=0$ for a planar surface, $m=1$ for a cylindrical surface, $m=2$ for a spherical surface; $j=1$ for linearly distributed fixed charges, $j=2$ for exponentially distributed fixed charges. Here, $a$ denotes the valence of the cation for an $a: b$

\begin{tabular}{|c|c|c|c|c|c|c|}
\hline \multirow{2}{*}{$\begin{array}{l}m \\
a \bigvee j\end{array}$} & \multicolumn{2}{|l|}{0} & \multicolumn{2}{|l|}{1} & \multicolumn{2}{|l|}{2} \\
\hline & 1 & 2 & 1 & 2 & 1 & 2 \\
\hline 1 & 3.6 & 2.4 & 2.6 & 4.1 & 2.2 & 3.4 \\
\hline 2 & 3.2 & 3.1 & 3.3 & 3.4 & 3.7 & 3.7 \\
\hline 3 & 4.2 & 2.8 & 4.3 & 3.5 & 4.2 & 4.3 \\
\hline
\end{tabular}
electrolyte

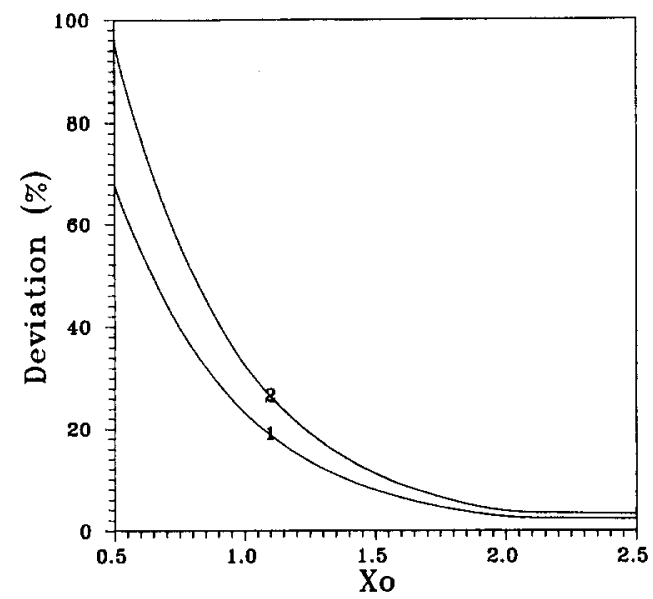

Fig. 5. The effect of the relative thickness of the uncharged core of a particle on the performance of the present approximate result. Key: same as Fig. 3, except that $X_{0} / d=2$ and $m=1$. Curve 1 , $(a: b)=(1: 2)$; Curve $2,(a: b)=(2: 1)$. The deviation is defined by $100 \% \times\left|\left(\Gamma_{\text {exact }}-\Gamma_{\text {approximate }}\right) / \Gamma_{\text {exact }}\right|$. 
It has been shown that for a planar surface with uniformly distributed fixed charges [3]

$X=k^{1 / 2} \sum_{i=1}^{\infty} a_{i}\left(Y-Y_{\text {Don }}\right)^{i}$

where $k=2+2 b / a$, and the coefficients $a_{i}, i=1,2, \ldots$ are functions of $\psi_{\mathrm{d}}$ and $\psi_{\mathrm{Don}}$. Substituting Eq. (25) into Eq. (24) and solving the resultant equation subject to Eq. (2d), we have the first-order approximation for $X=X(\psi)$. This relation can then be substituted into Eq. (24) to evaluate the second-order approximation. The procedure can be continued until an asymptotic expression is obtained.

If $\alpha \rightarrow 0$ and $m=0$, then $e_{i}=0, i=1,2, \ldots$ In this case, the set of equations (Eqs. $(7 \mathrm{a}),(7 \mathrm{~b}), \ldots)$ reduce to

$e_{0} e_{0}^{\prime}=(g+N) /(a+b)$

where $N=Z N_{0} N_{\mathrm{A}} / a n_{\mathrm{a}}^{0}$. The associated boundary condition is $e_{0} \rightarrow 0$ as $\psi \rightarrow \psi_{\mathrm{c}}$. Solving Eq. (26) gives

$(\mathrm{d} \psi / \mathrm{d} X)=-\operatorname{Sgn}(\psi)[2 /(a$

$$
\begin{aligned}
& +b)]^{1 / 2}\{(1 / b)[\exp (b \psi)-1] \\
& \left.+(1 / a)[\exp (-a \psi)-1]+N\left(\psi-\psi_{\mathrm{d}}\right)\right\}^{1 / 2}
\end{aligned}
$$

This expression is exactly the result for a planar surface with uniformly distributed fixed charges [3].

The effect of the distribution on fixed charges on the thermodynamic properties of the system under consideration is shown in Fig. 6. Here, the amount of ion adsorption is illustrated as an example. This figure reveals that, for the same apparent charge density, various fixed charge distributions yield different amount of ion adsorption. The greater the value of parameter $\alpha$ (the greater a distribution deviates from the uniform distribution), the greater the amount of ion adsorption deviates from the correct value. Apparently, assuming that fixed charges are distributed homogeneously may lead to an appreciable deviation.

\section{Conclusions}

In summary, the Poisson-Boltzmann equation governing the potential distribution of a general surface bearing an ion-penetrable membrane immersed in an arbitrary electrolyte solution is solved. The classic rigid surface model and symmetric electrolytes is extended

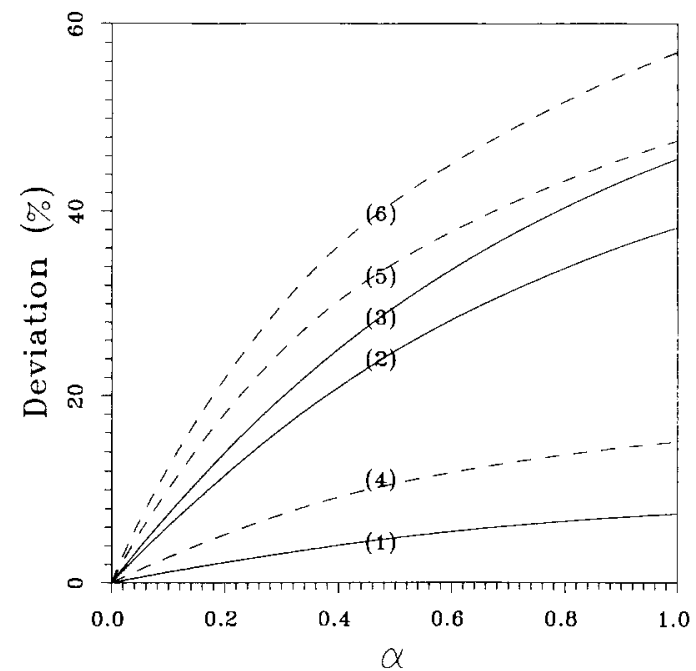

Fig. 6. The effect of the distribution of fixed charges on the amount of ion adsorption for the case $(a: b)=(1: 2)$. Solid lines denote linear fixed charge distribution, dashed lines represent nonlinear fixed charge distribution. Curves 1 and 4, planar surface; Curves 2 and 5 , cylindrical surface; Curves 3 and 6 , spherical surface. The apparent charge density in membrane is the same for all cases. Key: same as Fig. 3. The deviation is defined by $100 \% \mathrm{x}\left|\left(\Gamma_{\alpha \rightarrow 0}-\Gamma\right) / \Gamma_{\alpha \rightarrow 0}\right|$.

to the present general system. Explicit expressions for the basic thermodynamic properties are derived which are essential to the description of the nature of the system under consideration. In addition, we show quantitatively, that the distribution of fixed charges in the membrane phase can have a significant effect on its properties. This effect is either neglected, or stated qualitatively only, in the literature.

\section{List of symbols}

$a$

A

$b$

$d$

$d_{j, i}$

$e$

$e_{i}$

$F_{\mathrm{e} 1} \quad$ Helmholtz free energy $\left(\mathrm{J} / \mathrm{m}^{2}\right)$

$F_{\text {eld }} \quad$ Helmholtz free energy defined in Eq. (17a) $\left(\mathrm{J} / \mathrm{m}^{2}\right)$ 


\begin{tabular}{|c|c|}
\hline$F_{\mathrm{els}}$ & $\begin{array}{l}\text { Helmholtz free energy defined in Eq. } \\
(17 \mathrm{~b})\left(\mathrm{J} / \mathrm{m}^{2}\right)\end{array}$ \\
\hline$g$ & $\begin{array}{l}\text { function of electrostatic potential } \\
\text { (dimensionless) }\end{array}$ \\
\hline$H$ & derivative of potential (dimensionless) \\
\hline$i$ & region index $(0$ or 1$)$ \\
\hline$k$ & $=2+(2 b / a)$ \\
\hline$k_{1}, k_{2}$ & $\begin{array}{l}\text { parameters defined in Eq. (C15) } \\
\text { (dimensionless) }\end{array}$ \\
\hline$k_{3}$ & $\begin{array}{l}\text { parameter defined in Eq. (C15) } \\
\text { (dimensionless) }\end{array}$ \\
\hline$k_{\mathrm{B}}$ & Boltzmann constant $\left(\mathrm{J} /{ }^{\circ} \mathrm{K}\right)$ \\
\hline$m$ & $\begin{array}{l}\text { geometry parameter }(0,1 \text {, or } 2) \\
\text { (dimensionless) }\end{array}$ \\
\hline$n_{\mathrm{a}}^{0}$ & $\begin{array}{l}\text { number concentration of cations in bulk } \\
\text { liquid phase }\left(\text { no. } / \mathrm{m}^{3}\right)\end{array}$ \\
\hline$n_{i}$ & $\begin{array}{l}\text { number concentration of species } i \text { (no./ } \\
\mathrm{m}^{3} \text { ) }\end{array}$ \\
\hline$n_{i}^{0}$ & $\begin{array}{l}\text { number concentration of species } i \text { in } \\
\text { bulk liquid phase }\left(\text { no. } / \mathrm{m}^{2}\right)\end{array}$ \\
\hline$N(x)$ & dimensionless fixed charge distribution \\
\hline$N_{0}$ & average density of fixed charges (M) \\
\hline$N_{\mathrm{A}}$ & Avogadro number $(1 / \mathrm{mol})$ \\
\hline$N_{1}, N_{2}$ & dimensionless fixed charge distributions \\
\hline$p_{\mathrm{d} 1}$ & dimensionless interface charge density \\
\hline$q_{\mathrm{d} 1}$ & defined in Eq. (17e) (dimensionless) \\
\hline$r$ & distance $(\mathrm{m})$ \\
\hline$r_{0}$ & $\begin{array}{l}\text { position of core-membrane interface for } \\
\text { a planar geometry }(\mathrm{m})\end{array}$ \\
\hline $\overrightarrow{\mathbf{r}}$ & position vector $(\mathrm{m})$ \\
\hline$R_{1}, R_{2}$ & $\begin{array}{l}\text { defined in Eqs. (A1c) and (A1d) } \\
\text { (dimensionless) }\end{array}$ \\
\hline$S_{\mathrm{el}}$ & entropy of double layer $\left(\mathrm{J} / \mathrm{m}^{2} \mathrm{~K}\right)$ \\
\hline$T$ & absolute temperature $(\mathrm{K})$ \\
\hline$v$ & defined in Eq. (17e) (dimensionless) \\
\hline$V$ & volume of the system $\left(\mathrm{m}^{3}\right)$ \\
\hline$w_{j, i}$ & $\begin{array}{l}\text { coefficient defined in Eq. ( } 5 \text { ) } \\
\text { (dimensionless) }\end{array}$ \\
\hline$X$ & dimensionless position variable \\
\hline$X_{0}$ & $\begin{array}{l}\text { dimensionless size of the core of a } \\
\text { particle }\end{array}$ \\
\hline$Z$ & $\begin{array}{l}\text { valence of fixed charges } \\
\text { (dimensionless) }\end{array}$ \\
\hline
\end{tabular}

Greek letters

$\alpha$

$\Gamma$

\begin{tabular}{|c|c|}
\hline$\Gamma_{+}$ & $\begin{array}{l}\text { amount of adsorbed counter-ions ( } \mathrm{mol} / \\
\mathrm{m}^{2} \text { ) }\end{array}$ \\
\hline$\Gamma_{-}$ & amount of adsorbed co-ions $\left(\mathrm{mol} / \mathrm{m}^{2}\right)$ \\
\hline$\epsilon_{0}$ & permittivity of the vacuum $(\mathrm{C} / \mathrm{V} \mathrm{m})$ \\
\hline$\epsilon_{\mathrm{r}}$ & relative permittivity (dimensionless) \\
\hline$\mu_{+}$ & $\begin{array}{l}\text { chemical potential of counter-ions }(\mathrm{J} / \\
\mathrm{mol})\end{array}$ \\
\hline$\mu_{-}$ & chemical potential of co-ions $(\mathrm{J} / \mathrm{mol})$ \\
\hline$\sigma_{\mathrm{t}}$ & total apparent charge density $\left(\mathrm{C} / \mathrm{m}^{2}\right)$ \\
\hline$\sigma_{\mathrm{s} 1}$ & $\begin{array}{l}\text { equivalent charge density in membrane } \\
\left(\mathrm{C} / \mathrm{m}^{2}\right)\end{array}$ \\
\hline$\sigma_{\mathrm{d} 1}$ & $\begin{array}{l}\text { equivalent charge density at membrane- } \\
\text { liquid interface }\left(\mathrm{C} / \mathrm{m}^{2}\right)\end{array}$ \\
\hline$\kappa$ & reciprocal Debye length $(1 / \mathrm{m})$ \\
\hline$\psi$ & dimensionless electrostatic potential \\
\hline$\psi_{\mathrm{c}}$ & $\begin{array}{l}\text { dimensionless potential at core- } \\
\text { membrane interface }\end{array}$ \\
\hline$\psi_{\mathrm{d}}$ & $\begin{array}{l}\text { dimensionless potential at membrane- } \\
\text { liquid interface }\end{array}$ \\
\hline $\begin{array}{l}\psi_{\text {Don }} \\
\phi_{\mathrm{d}}\end{array}$ & $\begin{array}{l}\text { dimensionless Donnan potential } \\
\text { potential at membrane-liquid interface } \\
\text { (V) }\end{array}$ \\
\hline $\begin{array}{l}\phi_{\text {Don }} \\
\phi\end{array}$ & $\begin{array}{l}\text { Donnan potential }(\mathrm{V}) \\
\text { electrostatic potential }(\mathrm{V})\end{array}$ \\
\hline
\end{tabular}

Acknowledgements

This work is supported by the National Science Council of the Republic of China under Grant no. NSC84-2214-E-002-005.

\section{Appendix A}

The fixed charge distributions assumed, Eqs. (1) and (2), can be recast as

$$
\begin{aligned}
& N_{1}=S_{1}\left(Z N_{0} N_{\mathrm{A}} / a n_{a}^{0}\right) / R_{1}=M_{1} S_{1} \\
& N_{2}=S_{2}\left(Z N_{0} N_{\mathrm{A}} / a n_{a}^{0}\right) / R_{2}=M_{2} S_{2}
\end{aligned}
$$

where $M_{1}=\left(Z N_{0} N_{\mathrm{A}} / a n_{\mathrm{a}}^{0}\right) / R_{1}, \quad M_{2}=\left(Z N_{0} N_{\mathrm{A}} / a n_{\mathrm{a}}^{0}\right) /$ $R_{2}, \quad S_{1}=1+\alpha\left(X-X_{0}\right), \quad S_{2}=1+\exp \left[\alpha\left(X-X_{0}\right)\right]$. Here, $R_{1}$ and $R_{2}$ are defined by 


$$
\begin{aligned}
R_{1}= & \left\{\frac{\alpha}{m+2}\left[\left(X_{0}+d\right)^{m+2}-X_{0}^{m+2}\right]\right. \\
& \left.+\frac{1-\alpha X_{0}}{m+1}\left[\left(X_{0}+d\right)^{m+1}-X_{0}^{m+1}\right]\right\} / Q \quad(\mathrm{~A} 1 \mathrm{c}) \\
R_{2}= & \left\{\frac{1}{m+1}\left[\left(X_{0}+d\right)^{m+1}-X_{0}^{m+1}\right]+\sum_{i=0}^{m} \frac{(-1)^{m-i} m !}{\alpha^{(m-i+1)} i !}\right. \\
\left.\times\left[\left(X_{0}+d\right)^{i} \exp (\alpha d)-X_{0}^{i}\right]\right\} / Q & \quad \text { (A1d) } \\
Q= & d\left[X_{0}^{m}+(m / 2) X_{0}^{m-1} d\right] \quad \text { (A1e) }
\end{aligned}
$$

Eqs. (A1a) and (A1b) indicate that $N_{j}(X)$ increases with the dimensionless distance $X$ for a positive $\alpha$, which characterizes the variation in the distribution of fixed charges. This can easily be extended to the case that $N_{j}(X)$ decreases with $X$ through replacing $\left(X-X_{0}\right)$ in $S_{j}$ by $\left(X_{0}-X\right)$. Note that, since

$$
\kappa^{-1} \int_{X_{0}}^{X_{0}+d} N_{j} \mathrm{~d} X=\mathrm{d} \kappa^{-1}\left(Z N_{0} N_{\mathrm{A}} / a n_{a}^{0}\right)
$$

the dimensionless amount of fixed charges per unit area (apparent surface charge density, $\sigma_{\mathrm{t}}$ ) remains constant for a planar surface, and is independent of both parameters $\alpha$ and $j$. Similarly, it can be shown that this conclusion is also true for both cylindrical and spherical surfaces.

If $\alpha \rightarrow 0$, both $N_{1}$ and $N_{2}$ approach to $\left(Z N_{0} N_{\mathrm{A}} / a n_{\mathrm{a}}^{0}\right)$. In other words, both the linear and nonlinear distributions of fixed charges reduce to the same uniform distribution. On the other hand, if $\alpha \rightarrow \infty$,

$$
\begin{aligned}
& N_{1}\left(X=X_{0}\right) \rightarrow 0 \\
& N_{1}\left(X=X_{0}+d\right) \rightarrow 2\left(Z N_{0} N_{\mathrm{A}} / a n_{a}^{0}\right) \\
& N_{2}\left(X_{0} \leq X<X_{0}+d\right) \rightarrow 0 \\
& N_{2}\left(X=X_{0}+d\right) \rightarrow \infty
\end{aligned}
$$

By referring to Fig. 2, these expressions suggest that as $\alpha \rightarrow \infty, N_{1}$ increases linearly with $X$ from zero to twice of its average value, and $N_{2}$ approaches to a delta function, $\delta\left(X_{0}+d\right)$ at the outer boundary of the membrane.

Note that

$$
\lim _{\alpha \rightarrow 0} R_{1}=\frac{1}{m+1}\left[\left(X_{0}+d\right)^{m+1}-X_{0}^{m+1}\right] / Q, m=0,1,2
$$

$\lim _{a \rightarrow 0} R_{2}=\left\{\begin{array}{cc}2 & , m=0 \\ d^{2}\left[1+2\left(X_{0} / d\right) / Q(m=1)\right. & , m=1 \\ 2 d^{3}\left[(1 / 3)+\left(X_{0} / d\right)+\left(X_{0} / d\right)^{2}\right] / Q(m=2) & , m=2\end{array}\right.$

$\lim _{\alpha \rightarrow 0} S_{j}= \begin{cases}1, & j=1 \\ 2, & j=2\end{cases}$

Therefore if $\alpha \rightarrow 0$ (uniform fixed charge distribution), the value of $N_{j}$ corresponding to constant $\sigma_{\mathrm{t}}$ can be determined through Eqs. (1a), (1b), and (A7a)(A7c). It can be shown that $R_{1}=1$ and $R_{2}=2$ for $m=0$ and $m=1$, and if $\left(d / X_{0}\right) \ll 1, R_{1} \rightarrow 1$ and $R_{2} \rightarrow 2$ for $m=2$. Thus, if fixed charges are distributed uniformly, constant $N_{j}$ is equivalent to constant $\sigma_{t}$.

However, this is not true in general if the distribution of fixed charges is nonuniform. In this case, one can choose either constant $M$ (amont of fixed charges) or costant $\sigma_{t}$, but not both at the same time.

The asymptotic values of $N_{\mathrm{j}}$ as $\alpha \rightarrow \infty$ are

$$
\lim _{a \rightarrow \infty} N_{1}=\left\{\begin{array}{c}
2\left(X-X_{0}\right)\left(Z N_{0} N_{\mathrm{A}} / a n_{a}^{0}\right) / d, m=0 \\
\frac{6\left(X-X_{0}\right)}{d^{2}\left(3 X_{0}+2 d\right)}\left(Z N_{0} N_{\mathrm{A}} / a n_{a}^{0}\right) Q(m=1), m=1 \\
\frac{12\left(X-X_{0}\right)}{d^{2}\left(6 X_{0}^{2}+8 X_{0} d+3 d^{2}\right)}\left(Z N_{0} N_{\mathrm{A}} / a n_{a}^{0}\right) Q(m=2), m=2
\end{array}\right.
$$

$\lim _{\alpha \rightarrow \infty} N_{2}=\left\{\begin{array}{c}0, X_{0} \leq X<X_{0}+d \\ \infty, X=X_{0}+d\end{array}, m=0,1,2\right.$

Consider a differential volume of membrane $\delta V$. The corresponding differential area, $\delta A$, and the differential amount of fixed charges contained in $\delta V, \delta M$, are

$$
\begin{aligned}
& \delta A= \begin{cases}2 \pi L \kappa^{-2}\left(X_{0}+\delta X / 2\right) & \text { for cylinder } \\
4 \pi \kappa^{-2}\left(X_{0}+\delta X / 2\right)^{2} & \text { for sphere }\end{cases} \\
& \delta M= \begin{cases}2 \pi L Z e\left[N_{0} N_{\mathrm{A}} / R_{j}(m=1)\right] S_{j} \kappa^{-3} X \delta X & \text { for cylinder } \\
4 \pi Z e\left[N_{0} N_{\mathrm{A}} / R_{j}(m=2)\right] S_{j} \kappa^{-3} X^{2} \delta X & \text { for sphere }\end{cases}
\end{aligned}
$$

where $X$ represents a differential distance in the $r$ direction, and $L$ is the dimensionless length of a cylinder. The value of $\sigma_{t}$ can be calculated by 


$$
\delta_{\mathrm{t}}=\frac{\int_{X_{0}}^{X_{0}+d} \delta M}{\int_{X_{0}}^{X_{0}+d} \delta A}=Z e N_{0} N_{\mathrm{A}} d / \kappa, m=1 \text { or } 2
$$

Eqs. (A2) and (A12) suggest that the average or apparent density of fixed charges in membrane is independent of the shape of a particle.

\section{Appendix B}

The values of $w_{j, i}$ associated with Eq. (5) are

$$
\begin{array}{ll}
w_{1,0}=M_{1}(1+\alpha d / 2)+2 M_{1} \alpha\left(X_{0}+d / 2\right) & \text { (B1) } \\
w_{1,1}=-3 M_{1} \alpha\left(X_{0}+d / 2\right)^{2} & \text { (B2) } \\
w_{1,2}=M_{1} \alpha\left(X_{0}+d / 2\right)^{3} & \\
\ldots & \times\left[2+(\alpha / 2)\left(X_{0}+d / 2\right)\right] \\
w_{2,0}=M_{2}[\exp (d / 2)+1)+ & M_{2} \alpha \exp (d / 2)\left(X_{0}+d / 2\right) \\
& \times[2
\end{array}
$$

$w_{2,1}=-M_{2} \alpha \exp (d / 2)\left(X_{0}+d / 2\right)^{2}[3$

$$
\left.+\alpha\left(X_{0}+d / 2\right)\right]
$$

$w_{2,2}=M_{2} \alpha \exp (d / 2)\left(X_{0}+d / 2\right)^{3}[1$

$$
\left.+(\alpha / 2)\left(X_{0}+d / 2\right)\right]
$$

$\cdots$

\section{Appendix C}

Applying a similar procedure as that employed in the derivation of the result for a planar surface, we assume the following perturbation series:

$\mathrm{d} \psi / \mathrm{d} X=H(\psi, X)$

$$
=\sum_{i=0}^{n} c_{i}(\psi) / X^{i} \text { for double layer }
$$

$\mathrm{d} \psi / \mathrm{d} X=H(\psi, X)$

$$
=\sum_{i=0}^{n} d_{j, i}(\psi) / X^{i} \text { for membrane }
$$

Eq. (2) can be rewritten as

$$
\left(\frac{\partial H}{\partial X}\right)_{\psi}+H\left(\frac{\partial H}{\partial \psi}\right)_{X}+\frac{m H}{X}=\frac{g+i N_{j}}{a+b}
$$

The boundary conditions Eqs. (2a) through (2c) reduce to

$$
\begin{aligned}
H \rightarrow 0 \text { or } c_{i} \rightarrow 0 \text { as } \psi \rightarrow 0, i & =0,1,2, \ldots \\
H_{X \rightarrow\left(X_{0}+d\right)^{-}}=H_{X \rightarrow\left(X_{0}+d\right)^{+}} & \text {or } d_{j, i}=c_{i} \text { as } \psi \\
& \rightarrow \psi_{d}, i=0,1,2, \ldots
\end{aligned}
$$

Substituting Eq. (Cla) into Eq. (C2) and collecting terms of the same order in $X$ lead to a set of equations in $c_{i}$. Solving these equations successively subject to Eq. (C3) yields Eq. (11). Similarly, substituting Eq. (C1b) into Eq. (C2), employing Eq. (5), and collecting terms of the same order in $X$ lead to a set of equations in $d_{j, i}$. The first three of these equations are

$$
\begin{aligned}
& d_{j, 0} d_{j, 0}^{\prime}=\left(g+w_{j, 0}\right) /(a+b) \\
& d_{j, 0} d_{j, 1}^{\prime}+d_{j, 1} d_{j, 0}^{\prime}+m d_{j, 0}=w_{j, 1} /(a+b) \\
& d_{j, 0} d_{j, 2}^{\prime}+d_{j, 1} d_{j, 1}^{\prime}+d_{j, 2} d_{j, 0}^{\prime}+(m
\end{aligned}
$$

$$
-1) d_{j, 1}=w_{j, 2} /(a+b)
$$

Expanding $\left(d_{j, 0} d_{j, 1}\right)^{\prime}$ and $\left(d_{j, 0} d_{j, 2}\right)^{\prime}$, where the differentiation is with respect to $\psi$, into power series of $Y$ around $Y_{\mathrm{av}}$, Eqs. (C6) and (C7) become, respectively,

$\frac{w_{j, 1}}{a+b}-m d_{j, 0}=\sum_{i=0}^{n_{1}} u_{j, i} Y^{i}$

and

$\frac{w_{j, 2}}{a+b}-d_{j, 1} d_{j, 1}^{\prime}-(m-1) d_{j, 1}=\sum_{i=0}^{n_{2}} v_{j, i} Y^{i}$

where $Y=\exp (a \psi / 2), Y_{\mathrm{av}}=\left(Y_{\mathrm{c}}+Y_{\mathrm{d}}\right) / 2, Y_{\mathrm{c}}$ and $Y_{\mathrm{d}}$ being the value of $Y$ evaluated at $\psi_{\mathrm{c}}$ and $\psi_{\mathrm{d}}$, respectively, and

$$
\begin{aligned}
& u_{j, 0}=\frac{w_{j, 1}}{a+b} \\
& -m\left[d_{j, 0, \mathrm{av}}-Y_{\mathrm{av}} d_{j, 0, \mathrm{av}}^{\prime}+\left(Y_{\mathrm{av}}^{2} / 2\right) d_{j, 0, \mathrm{av}}^{\prime \prime}-\left(Y_{\mathrm{av}}^{3} / 6\right) d_{j, 0, \mathrm{av}}^{\prime \prime \prime}\right]
\end{aligned}
$$

$$
u_{j, 1}=-m\left[d_{j, 0, \mathrm{av}}^{\prime}-Y_{\mathrm{av}} d_{j, 0, \mathrm{av}}^{\prime \prime}+\left(Y_{\mathrm{av}}^{2} / 2\right) d_{j, 0, \mathrm{av}}^{\prime \prime \prime}\right]
$$

(C10b) 
$u_{j, 2}=-m\left[(1 / 2) d_{j, 0, \mathrm{av}}^{\prime \prime}-\left(Y_{\mathrm{av}} / 2\right) d_{j, 0, \mathrm{av}}^{\prime \prime \prime}\right.$

$(\mathrm{C} 10 \mathrm{c})$

$u_{j, 3}=-m(1 / 6) d_{j, 0, \mathrm{av}}^{\prime \prime \prime}$

(C10d)

$\cdots$

$v_{j, 0}=\frac{w_{j, 2}}{a+b}-\left[d_{j, 1, \mathrm{av}} d_{j, 1, \mathrm{av}}^{\prime}+(m-1) d_{j, 1, \mathrm{av}}\right]$

$+\left[d_{j, 1, \mathrm{av}} d_{j, 1, \mathrm{av}}^{\prime \prime}+\left(d_{j, 1, \mathrm{av}}^{\prime}\right)^{2}+(m\right.$

$\left.-1) d_{j, 1, \mathrm{av}}^{\prime}\right] Y_{\mathrm{av}}-\left[d_{j, 1, \mathrm{av}} d_{j, 1, \mathrm{av}}^{\prime \prime \prime}+3 d_{j, 1, \mathrm{av}}^{\prime} d_{j, 1, \mathrm{av}}^{\prime \prime}+(m-1)\right.$

$\left.\times d_{j, 1, \mathrm{av}}^{\prime \prime}\right] Y_{\mathrm{av}}^{2}$

(C10e)

$v_{j, 1}=-\left[d_{j, 1, \mathrm{av}} d_{j, 1, \mathrm{av}}^{\prime \prime}+\left(d_{j, 1, \mathrm{av}}^{\prime}\right)^{2}+(m-1) d_{j, 1, \mathrm{av}}^{\prime}\right]$

$+2\left[d_{j, 1, \mathrm{av}} d_{j, 1, \mathrm{av}}^{\prime \prime \prime}+3 d_{j, 1, \mathrm{av}}^{\prime} d_{j, 1, \mathrm{av}}^{\prime \prime}+(m-1) \times d_{j, 1, \mathrm{av}}^{\prime \prime}\right] Y_{\mathrm{av}}$

(C10f)

$v_{j, 2}=-\left[d_{j, 1, \mathrm{av}} d_{j, 1, \mathrm{av}}^{\prime \prime \prime}+3 d_{j, 1, \mathrm{av}}^{\prime} d_{j, 1, \mathrm{av}}^{\prime \prime}+(m-1) d_{j, 1, \mathrm{av}}^{\prime \prime}\right]$

$(\mathrm{C} 10 \mathrm{~g})$

$\cdots$

In these expressions, $d_{j, 0, \mathrm{av}}, d_{j, 0, \mathrm{av}}{ }^{\prime}, d_{j, 0, \mathrm{av}}{ }^{\prime \prime}$, and $d_{j, 0, \mathrm{av}}{ }^{\prime \prime \prime}$ are, respectively, $d_{j, 0}$, its first, second, and third derivatives with respect to $Y$ evaluated at $Y_{\mathrm{av}}$, and $d_{j, 1, \mathrm{av}}$, $d_{j, 1, \mathrm{av}}{ }^{\prime}, d_{j, 1, \mathrm{av}}{ }^{\prime \prime}$, and $d_{j, 1, \mathrm{av}}{ }^{\prime \prime \prime}$ are, respectively, $d_{j, 1}$, its first, second, and third derivatives with respect to $Y$ evaluated at $Y_{\mathrm{av}}$. Solving Eq. (C5) subject to Eq. (C4) gives

$d_{j, 0}=[2 /(a+b)]^{1 / 2}\{(1 / b)[\exp (b \psi)-1]$

$+(1 / a)[\exp (-a \psi)-1]+w_{j, 0}(\psi$

$\left.\left.-\psi_{d}\right)\right\}^{1 / 2}=(2 / a Y)\left(f_{j, 1} / k\right)^{1 / 2}$

where

$f_{j, 1}=1-\frac{k}{k-2} Y^{2}+\frac{2}{k-2} Y^{k}+2 w_{j, 0} Y^{2} \ln \left(Y / Y_{d}\right)$

Substituting this expression and (C8) into Eq. (C6) and solving the resultant expression subject to $\mathrm{Eq}$. (C4), we obtain

$$
\begin{array}{r}
d_{j, 1}=\frac{2}{a\left(f_{j, 1}^{1 / 2} / Y\right)}\left[u_{j, 0} \ln \left(\frac{y}{Y_{d}}\right)+\sum_{i=1}^{n_{1}} \frac{u_{j, i}}{i}\left(Y^{i}-Y_{d}^{i}\right)\right. \\
\left.+\frac{2 m k_{3}}{a}\left(\frac{Y_{d}+Y_{d}^{-1}-2}{2}\right)\right]
\end{array}
$$

Substituting Eqs. (C11), (C13), and (C9) into Eq. (C7) and solving the resultant equation subject to Eq. (C4) yield

$$
\begin{aligned}
d_{j, 2}= & \frac{2}{a\left(f_{j, 1}^{1 / 2} / Y\right)}\left\{v_{j, 0} \ln \left(\frac{Y}{Y_{d}}\right)+\sum_{i=1}^{n_{2}} \frac{v_{j, i}}{i}\left(Y^{i}-Y_{d}^{i}\right)\right. \\
& \left.+\frac{\left[m\left(Y_{d}-1\right) /\left(Y_{d}+1\right)\right]^{2}-4 m(m-1) \ln \left[\left(Y_{d}^{1 / 2}-Y_{d}^{-1 / 2}\right) / 2\right]}{k_{3}\left(Y_{d}-Y_{d}^{-1}\right)}\right\}
\end{aligned}
$$

In these expressions,

$k_{3}= \begin{cases}{\left[(k-2) k_{1}+2 k_{2}\right] / k,} & \text { if } k \leq 4 \\ {\left[2 k_{1}+(k-2) k_{2}\right] / k,} & \text { if } k>4\end{cases}$

where $k_{1}=2 /\left\{k^{1 / 2}\left[(k / 2)^{2 /(k-2)}-1\right]\right\}, k_{2}=2 / k^{1 / 2}$, and $k=2+2 b / a$. The parameter $k$ is a measure of the degree of asymmetry of the electrolyte.

\section{Appendix D}

As a first approximation, Eq. (13) is solved by neglecting $d_{1}, d_{2}, \ldots$ etc. The first-order approximate solution thus obtained, $\psi_{d, 1}$ is

$$
\begin{aligned}
\psi_{a, 1}=\psi_{\text {Don }}+\left(1 / w_{j, 0}\right)\left\{( 1 / b ) \left[\exp \left(b \psi_{\text {Don }}\right)\right.\right. \\
\left.-1]+(1 / a)\left[\exp \left(-a \psi_{\text {Don }}\right)-1\right]\right\}
\end{aligned}
$$

Substituting Eq. (D1) into the second term on the lefthand side of Eq. (13), neglecting $d_{2}, d_{3}, \ldots$ etc., and solving the resultant equation, we obtain the secondorder approximate solution $\psi_{d .2}$ as

$$
\begin{aligned}
& \psi_{d .2}=\psi_{\text {Don }}+\left(1 / w_{j .0}\right)\left\{( 1 / b ) \left[\exp \left(b \psi_{\text {Don }}\right)\right.\right. \\
& -1]+(1 / a)\left[\exp \left(-a \psi_{\text {Don }}\right)-1\right]-[(a \\
& \left.+b) / 2]\left[d_{1}\left(\psi_{\text {Don }}\right)_{1} / X_{0}\right]^{2}\right\}
\end{aligned}
$$

where $d_{1}\left(\psi_{\text {Don }}\right)_{1}$ is the value of $d_{1}\left(\psi_{\text {Don }}\right)$ with $\psi_{d}$ replaced by $\psi_{d, 1}$. Substituting Eq. (D2) into $d_{1}$ and $d_{2}$ in Eq. (13), neglecting $d_{3}, d_{4}, \ldots$ etc., and solving the resultant equation, we have the third-order approximate solution $\psi_{d, 3}$ as

$$
\begin{aligned}
& \psi_{d} \simeq \psi_{d, 3}=\psi_{\text {Don }}+\left(1 / w_{j, 0}\right)\left\{(1 / b)\left[\exp \left(b \psi_{\text {Don }}\right)-1\right]\right. \\
& \left.\left.+(1 / a)\left[\exp \left(-a \psi_{\text {Don }}\right)-1\right)\right]\right\}-[(a \\
& +b) / 2]\left[\frac{d_{1}\left(\psi_{\text {Don }}\right)_{2}}{X_{0}}+\frac{d_{2}\left(\psi_{\text {Don }}\right)_{2}}{X_{0}^{2}}\right]^{2}
\end{aligned}
$$

(D3) 
where $d_{1}\left(\psi_{\text {Don }}\right)_{2}$ and $d_{2}\left(\psi_{\text {Don }}\right)_{2}$ are, respectively, the values of $d_{1}\left(\psi_{\text {Don }}\right)$ and $d_{2}\left(\psi_{\text {Don }}\right)$ with $\psi_{d}$ replaced by $\psi_{d, 2}$.

\section{Appendix E}

The value of $D_{d 1}$ in Eq. (19) and the relevant properties are defined by

$$
\begin{aligned}
& D_{d 1}=p_{d 1}+1 \\
& -q_{d 1}+\frac{1}{\left(X_{0}+d\right)} \frac{m}{k_{3}} \frac{q_{d 1}-1}{q_{d 1}}+\frac{1}{\left(X_{0}+d\right)^{2}} \times\left\{\frac{m^{2}}{4 k_{3}^{2}}[-1\right. \\
& \left.-\frac{2}{q_{d 1}\left(q_{d 1}+1\right)}+\frac{2}{q_{d 1}^{3}}\right]+\frac{m(m-1)}{2 k_{3}^{2}}\left[\frac{2}{q_{d 1}} \ln \left(\frac{q_{d 1}+1}{2}\right)\right. \\
& \left.\left.+\int_{v}^{1} \frac{\ln Z_{1}}{Z_{1}-1} d Z_{1}\right]\right\}
\end{aligned}
$$

$$
\begin{aligned}
& \frac{\partial F_{\mathrm{e} l \mathrm{~s}}}{\partial \kappa} \\
& =d \kappa^{-1} k_{\mathrm{B}} T \frac{\partial n_{a}^{0}}{\partial \kappa}\left[2 \ln Y_{\text {Don }}+Y_{\text {Don }}^{-2}+\frac{2}{k-2} Y_{\text {Don }}-Y_{\text {Don }}^{\prime 2}\right. \\
& \left.-\frac{2}{k-2} Y_{\text {Don }}^{k-2}\right] \\
& +d \kappa^{-1} k_{\mathrm{B}} T n_{a}^{0}\left[\left(\frac{1}{2} Y_{\text {Don }}^{-1}-2 Y_{\text {Don }}^{-3}+2 Y_{\text {Don }}^{k-3}\right)\right. \\
& \left.\times \frac{\partial Y_{\text {Don }}}{\partial \kappa}+\left(2 Y_{\text {Don }}^{\prime-3}-2 Y_{\text {Don }}^{k-3}\right) \frac{\partial Y_{\text {Don }}^{\prime}}{\partial \kappa}\right]-\frac{2 \sigma_{d 1} k_{\mathrm{B}} T}{a e} \\
& \times Y_{\text {Don }}^{\prime} \frac{\partial Y_{\text {Don }}^{\prime}}{\partial \kappa} \\
& \partial n_{a}^{0} / \partial \kappa=2 n_{a}^{0} / \kappa \\
& \frac{\partial Y_{\text {Don }}}{\partial \kappa}=\frac{-Y_{\text {Don }}^{2}}{k Y_{\text {Don }}^{-1}+2 N_{j}\left(X_{0}\right) Y_{\text {Don }}} \frac{\partial N_{j}\left(X_{0}\right)}{\partial \kappa} \\
& \frac{\partial Y_{\text {Don }}^{\prime}}{\partial \kappa}=\frac{-Y_{\text {Don }}^{\prime 2}}{k Y_{\text {Don }}^{\prime k-1}+2 N_{j}\left(X_{0}\right)^{\prime} Y_{\text {Don }}^{\prime}} \frac{\partial N_{j}\left(X_{0}\right)^{\prime}}{\partial \kappa}
\end{aligned}
$$

$$
\begin{aligned}
& \partial N_{j}\left(X_{0}\right) / \partial \kappa=-2 N_{j}\left(X_{0}\right) / \kappa \\
& \partial N_{j}\left(X_{0}\right)^{\prime} / \partial \kappa=-2 N_{j}\left(X_{0}\right)^{\prime} / \kappa \\
& \frac{N_{j}\left(X_{0}\right)^{\prime}}{N_{j}\left(X_{0}\right)}=\frac{N_{0}^{\prime}}{N_{0}}=\frac{\sigma_{d 1}}{\sigma_{\mathrm{t}}} \frac{2 k_{3} \epsilon_{0} \epsilon_{\mathrm{r}} k_{\mathrm{B}} T \kappa \sinh \left(a \psi_{d} / 2\right)}{-Z e^{2} N_{0} N_{\mathrm{A}} a d / \kappa}
\end{aligned}
$$

The reference state for the Helmholtz free energy is chosen as the state of an uncharged surface. Here, $N_{0}^{\prime}$ is the average fixed space charge density defined by $N_{0} \sigma_{d 1} / \sigma_{t}$, and $N_{j}\left(X_{0}\right)^{\prime}$ is the value of $N_{j}\left(X_{0}\right)$ based on $N_{0}^{\prime}$.

\section{References}

[1] R.J. Hunter, Fundations of Colloid Science, Vol. I, Clarendon, Oxford, 1987.

[2] J.P. Hsu and Y.C. Kuo, Approximate analytical expressions for the properties of an electrical double layer with asymmetric electrolytes, J. Chem. Soc., Faraday Trans., 89 (1993) 1229.

[3] J.P. Hsu and Y.C. Kuo, Approximate analytical expressions for the properties of a double layer with asymmetric electrolytes: ion-penetrable charged membranes, J. Colloid Interface Sci., 166 ( 1994) 208.

[4] J.H. Petropoulos, Membrane transport properties in relation to microscopic and macroscopic structural inhomogeneity, J. Membrane Sci., 52 (1990) 305.

[5] K.A. Mauritz and A.J. Hopfinger, Structural properties of membrane ionomers, in J.O'M. Bockris, B.E. Conway and R.E. White (Eds.), Modern Aspects of Electrochemistry, Plenum, New York, NY, 1982.

[6] M. Pineri, Microstructure of organic ionic membranes, ACS Symp. Ser., 302 (1986) 159.

[7] H. Reiss and I.C. Bassignana, Critique of the mechanism of superselectivity in ion exchange membranes, J. Membrane Sci., 11 (1982) 219.

[8] C. Selvey and H. Reiss, Ion transport in inhomogeneous ion exchange membranes, J. Membrane Sci., 23 ( 1985) 11.

[9] J.P. Hsu, W.C. Hsu and Y.I. Chang, Effects of fixed-charge distribution and $\mathrm{pH}$ on the electrophoretic mobility of biological cells, Colloid Polym. Sci., 272 (1994) 251.

[10] J.Th.G. Overbeek, G.J. Verhoeckx, P.L. de Bruyn and H.N.W. Lekkerkerker, On understanding microemulsions II. Thermodynamics of droplet-type microemulsions, J. Colloid Interface Sci., 119 (1987) 422.

[11] J.P. Hsu and Y.C. Kuo, Approximate analytical expressions for the properties of an electrical double layer with asymmetric electrolytes: cylindrical and spherical geometries, J. Colloid Interface Sci., 167 ( 1994$) 35$.

[12] H.G.L. Coster, The double fixed charge membrane solutionmembrane ion partition effects and membrane potentials, Biophys. J., 13 (1973) 133. 